\title{
Necessidade de Cuidados Intensivos em Maternidade Pública Terciária
}

\author{
Necessity of Intensive Care in a Tertiary Public Maternity Hospital
}

Marcello Braga Viggiano ${ }^{1,2}$, Mauricio Guilherme Campos Viggiano ${ }^{1}$, Eduardo de Souza ${ }^{2}$, Luiz Camano ${ }^{2}$

\section{RESUM0}

Objetivos: avaliar os aspectos epidemiológicos e os relacionados ao parto de gestantes $e$ puérperas transferidas para unidades de terapia intensiva (UTI’s) e a freqüência com que estas pacientes necessitam de cuidados intensivos.

Métodos: estudo observacional e descritivo das transferências obstétricas para UTI's, entre janeiro de 1999 e dezembro de 2001. A análise incluiu as seguintes variáveis: idade materna, paridade, indicações obstétricas e não-obstétricas para as transferências, momento em que estas ocorrem no ciclo gravídico-puerperal, tipo de parto, desfecho materno e freqüência com que estas transferências ocorrem em relação ao número total de partos (razão de morte iminente-RMI). A análise estatistica foi realizada pelo teste do $\chi^{2}$ ou teste exato de Fisher, com nivel de significância fixado em $5 \%$.

Resultados: no periodo de 36 meses, ocorreram 86 transferências maternas (em 4.560 partos). Entre as pacientes transferidas, 52,3\% (n=45) eram nuliparas e $63(73,2 \%)$ tinham idade entre 19 e 35 anos. As síndromes hipertensivas representaram 57,7\% (n=41) das indicações $e$ as síndromes hemorrágicas, 19,7\% $(n=14)$. Eclâmpsia $(n=23)$, síndrome HELLP $(n=13) e$ descolamento prematuro da placenta normalmente inserida $(\mathrm{n}=5)$ foram as causas obstétricas mais prevalentes na determinação destas transferências. As cardiopatias maternas somaram 4 casos entre as indicações não-obstétricas. Houve predomínio das transferências puerperais (82,35\%). Cinqüenta e cinco pacientes $(72,3 \%)$ tiveram seus partos realizados através de cesarianas. O tempo médio de internação nas UTI’s foi 5,1 dias. A mortalidade materna encontrada neste estudo correspondeu a $24,2 \%$, sendo que as sindromes hipertensivas foram responsáveis por 52,9\% (9/17) das mortes obstétricas diretas. Não houve diferença significante $(p=0,81)$ entre os decessos maternos e suas causas (sindromes hipertensivas, hemorrágicas, infecciosas ou outras) ou entre mortalidade materna e duração da internação (< ou >48 horas) nas UTI's ( $p=0,08$ ). A RMI encontrada foi de 18,8/1.000 partos.

Conclusões: a necessidade de cuidados intensivos estimada pela RMI foi de 18,8/1.000 partos, sendo que as síndromes hipertensivas induzidas pela gestação foram responsáveis pela maioria das indicações para as transferências maternas.

PALAVRAS-CHAVE: Cuidados intensivos. Hipertensão arterial. Complicações da gravidez. Mortalidade materna

${ }^{1}$ Serviço de Obstetrícia do Hospital Materno Infantil de Goiânia - GO

${ }^{2}$ Departamento de Obstetrícia da Universidade Federal de São Paulo - Escola Paulista de Medicina (UNIFESP-EPM) Correspondência:

Marcello Viggiano

Avenida 5 $5^{\mathrm{a}}$ Radial 423, apto. 302 - Setor Bela Vista

74823-030 - Goiânia - GO

Fone: (62) 255-7871

e-mail: marcelloviggiano@ig.com.br

Apoio financeiro: CAPES (Coordenação de Aperfeiçoamento de Pessoal de Nivel Superior)

\section{Introdução}

A situação humana e social de um país e de seu povo é, certamente, expressa pela mortalidade materna ${ }^{1}$. A Organização Mundial da Saúde (OMS) e a Federação Internacional de Ginecologia e Obstetrícia (FIGO) definem mortalidade materna como sendo o óbito da mulher enquanto grávida ou dentro dos 42 dias completos do puerpério, indepen- 
dente da duração da prenhez e do sítio de implantação do ovo, decesso proveniente de qualquer causa relacionada ou agravada pela gestação, ou de seu tratamento, excluindo-se fatores acidentais ${ }^{2}$. Considerando-se que pelo menos 500.000 óbitos maternos ocorrem, anualmente, no mundo todo ${ }^{1}$, os casos nos quais as gestantes ou as puérperas apresentam disfunções sistêmicas agudas, que, se não tratadas apropriadamente, poderiam resultar na morte das mesmas, despontam como indicadores essenciais na avaliação das principais e mais graves doenças obstétricas, freqüentemente relacionadas à mortalidade materna.

Escassa literatura existe sobre a necessidade de cuidados intensivos ou transferências para unidades de terapia intensiva em Obstetrícia. Mabie e Sibai ${ }^{3}$, em 1990, descreveram recursos de aparelhagem técnica, fundamentais nestes tipos especiais de unidades. Os mesmos autores ressaltaram ainda os benefícios específicos das unidades de terapia intensiva obstétricas, como a melhoria da assistência às pacientes, uma vez que observação rigorosa e organização possibilitariam não só a detecção precoce de doenças intercorrentes, mas também a profilaxia de suas complicações.

Por outro lado, Mantel et $a .^{4}$ revelaram que o benefício principal destas unidades de cuidados intensivos específicos para os médicos assistentes seria uma visão mais apurada dos padrões destas doenças graves e por meio destes, idéias mais relevantes sobre as necessidades dos programas preventivos que poderiam ser criados. Embora relativamente incomuns, as doenças graves, induzidas ou coincidentes com as gestações, podem requerer cuidados intensivos especializados para algumas pacientes, e representam desafios quanto ao diagnóstico e terapêutica utilizada. O equilíbrio entre o bem-estar materno e o fetal, no entanto, freqüentemente representa dilemas médicos e éticos peculiares ${ }^{5}$.

Atualmente, sabe-se que as áreas com os maiores índices de mortalidade materna requerem as mais urgentes intervenções, mas, inevitavelmente, estas são as regiões que menos levantamentos e estudos confiáveis fornecem sobre o assunto ${ }^{4}$. Nas regiões onde a cobertura assistencial dos serviços de saúde é pequena, as pesquisas e os estudos são os únicos meios para obtenção de dados populacionais sobre a prevalência de certas morbidades ${ }^{4}$ O Brasil ocupa lugar relativamente modesto nessa hecatombe assistencial feminina, ostentando coeficiente de 55,7 óbitos maternos por 100.000 nascidos vivos, consoante dados do Ministério da Saúde de $1999^{6}$. A difusão da assistência intensiva, especificamente obstétrica, ainda é utópica em nosso país, en- tretanto movimentos e discussões iniciais para a criação das mesmas já são realidade em certas instituições de ensino, em algumas regiões do Brasil. O que mais comumente observamos é a generalização da assistência intensiva por profissionais não especializados em intercorrências durante o ciclo gravídico-puerperal.

Em razão de cada óbito materno mascarar diversas intercorrências de doenças ou complicações, a atenção está sendo voltada para a morbidade propriamente dita, para que esta assegure informações importantes para os programas sobre maternidade segura. Pouca informação existe sobre os eventos maternos não-fatais, e estes podem representar diferentes padrões de intercorrências ou responder às intervenções de maneira diversa dos casos que resultam nos óbitos maternos ${ }^{4}$.

Baseado no exposto, pela grande importância que o tema representa, a pesquisa que se segue oferece dados sobre os maiores desafios e complicações na nossa prática contemporânea, procurando, desta forma, avaliar a necessidade de cuidados intensivos em Obstetrícia.

\section{Pacientes e Métodos}

Os dados presentes neste trabalho do Serviço de Obstetrícia do Hospital Materno Infantil de Goiânia (HMI-GO) são fruto de estudo longitudinal, retrospectivo, descritivo, desenvolvido no período de três anos, de janeiro de 1999 a dezembro de 2001 , incluindo todas as transferências de gestantes e puérperas para centros de terapia intensiva, bem como o número total de partos, abortamentos, gestações ectópicas e molas hidatiformes.

O HMI-GO é constituído por maternidade pública (conveniada ao Sistema Único de Saúde SUS) com 35 leitos distribuídos em seis enfermarias, onde dois destes são considerados como unidades intermediárias ou semi-intensivas, de nível terciário, vinculada à Faculdade de Medicina da Universidade Federal de Goiás (UFG-GO) e à residência médica em Ginecologia e Obstetrícia. Sendo referência para gestações de alto risco, e atendendo todo o Estado de Goiás e estados vizinhos, apresenta média de 4.500 internações e 1.500 partos por ano.

O Hospital Materno Infantil de Goiânia valese de três unidades de terapia intensiva externas, separadas geograficamente, porém semelhantes sob o ponto de vista estrutural e também profissionalmente, no que diz respeito ao corpo clínico uma vez que, no período estudado, o centro de terapia intensiva materna do próprio hospital 
encontrava-se, ainda, em fase final de construção. Estas unidades distam do hospital cerca de dez quilômetros. A transferência depende da disponibilidade de vagas.

No universo de 4.560 partos, 3.051 abortamentos, 172 gestações ectópicas e 153 casos de molas hidatiformes, ocorridos no período estudado de três anos, houve 86 episódios em que gestantes ou puérperas tiveram indicações de transferências para unidades de terapia intensiva. Dentre as variáveis por nós apreciadas, alguns aspectos conceituais devem ser analisados, como por exemplo em relação à idade das pacientes, já que esta foi subdividida em três grupos principais.

O grupo $\mathrm{G}<19$ incluiu as pacientes com idade inferior a 19 anos completos; o grupo G 19-35 foi formado por pacientes na faixa etária entre 19 e 35 anos completos, e o grupo $\mathrm{G}>35$ por aquelas com mais de 35 anos. Em relação à paridade, consideramos como nulíparas as mulheres que nunca haviam parido, primíparas seriam as que já haviam parido uma vez e multíparas aquelas que o haviam feito mais de uma vez.

Os diagnósticos e as indicações para as transferências das pacientes foram estabelecidos pelo corpo clínico presente nos plantões, em conjunto com docentes, por meio de propedêutica clínica e/ou subsidiária. O momento em que estas transferências ocorreram, com a gravidez ainda em curso, foi calculado em semanas, baseandose em datas de última menstruação confiáveis ou em ultra-sonografias precoces realizadas até a vigésima semana de gestação ${ }^{7}$. O momento da transferência foi analisado em relação à parturição, sendo os dados expressos em dias, antes ou após a efetuação da mesma.

Consideramos os partos vaginais como podendo ser espontâneos ou induzidos. Por outro lado, os partos cesáreos foram subdivididos em duas categorias, podendo ser realizados de maneira eletiva ou não eletiva ${ }^{8}$. Avaliamos os desfechos maternos através dos dias de internação nas unidades de terapia intensiva, sendo os óbitos analisados conforme as causas e modos de apuração, como por exemplo através de propedêutica clínica realizada pelos médicos plantonistas, exames subsidiários ou necrópsia. Analisamos a freqüência com que as pacientes obstétricas foram encaminhadas às UTI's pela relação entre o número de transferências e o número total de partos ocorridos durante o período estudado. Denominamos originalmente e a partir de agora denominaremos esta freqüência por razão de morte iminente (RMI), a qual será calculado, num determinado intervalo de tempo, em relação a 1.000 partos.

A análise estatística foi realizada com pro- grama de domínio público "STATA" versão 7.0, da SPSS, Inc. (USA), utilizando-se os testes $\chi^{2}$ e o teste exato de Fisher, se necessário, considerandose significativo um erro a menor que 5\%.

O projeto de pesquisa foi previamente analisado e aprovado pelas Comissões de Ética em Pesquisa do HMI-GO e da UNIFESP-EPM.

\section{Resultados}

Durante os três anos de pesquisa, houve 86 situações em que as pacientes necessitaram de acompanhamento intensivo, sendo que no ano de 1999 ocorreram 32 transferências, o que representou 37,2\% de todas elas. Em 2000, foram 23 (26,7\%), e no ano seguinte, 31 (36,1\%) casos.

Em relação à idade das pacientes transferidas, das 86 pacientes transferidas, 63 (73,3\%) pertenciam ao grupo 19-35, sendo que 18 (20,9\%) eram do grupo $<19$ e outras $5(5,8 \%)$ do grupo $>35$. No geral, considerando-se os três grupos avaliados, encontramos idade média de 24,6 anos, com mediana de 24 e desvio padrão de 5,9. A idade mínima foi de 14 e a máxima de 40 anos. Quanto a paridade das gestantes transferidas, percebemos que as nuliparas representaram $52,3 \%$ dos casos $(n=45)$, ao passo que as primiparas e multiparas corresponderam a 23,3\% $(n=20)$ e 24,4\% (n=21), respectivamente.

As causas obstétricas responderam por $82,6 \%$ das indicações para as transferências, sendo que as não obstétricas determinaram 17,4\% das mesmas. Assim, apuramos que os distúrbios hipertensivos induzidos pela gestação representaram mais da metade $(57,7 \%)$ das indicações obstétricas para as transferências para as UTI’s, tendo as doenças hemorrágicas representado 19,7\% da nossa amostra e as doenças infecciosas, 9,8\% (Tabela 1). Considerando-se as indicações específicas para as transferências, percebemos que a eclâmpsia e a síndrome HELLP destacam-se dentre as doenças hipertensivas, representando 56,1 e 31,7\%, respectivamente (Figura 1). Em relação às doenças hemorrágicas, destacaram-se o descolamento prematuro da placenta normalmente inserida $(35,8 \%)$ e o acretismo placentário $(28,6 \%)$ como indicações principais. Representaram parcela neste contexto, um caso de hemorragia após abortamento, um de rotura uterina e três casos caracterizados por coagulopatia intravascular disseminada (CIVD).

Nota-se que dentre as doenças infecciosas, aquelas que aconteciam após abortamento representaram 57,1\% dos casos (4/7), ao passo que as puerperais corresponderam a 42,8\% (3/7). 
Tabela 1 - Número e porcentagem de pacientes transferidas do Hospital Materno Infantil para as unidades de terapia intensiva, segundo indicações obstétricas.

\begin{tabular}{lcr}
\hline Indicações & $\mathbf{n}$ & $\%$ \\
\hline Doença hipertensiva & 41 & 57,7 \\
Doença hemorrágica & 14 & 19,7 \\
Doença infecciosa & 7 & 9,8 \\
Outras & 9 & 12,6 \\
Total & 71 & 100 \\
\hline
\end{tabular}

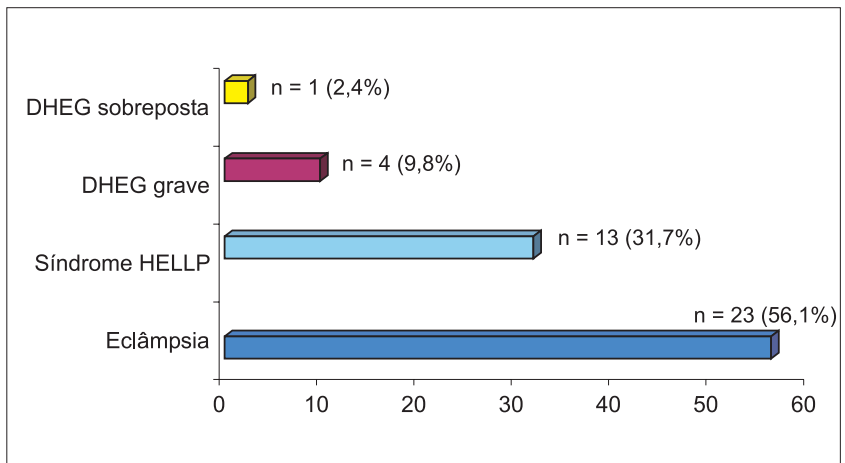

DHEG: Doença hipertensiva específica da gravidez.

HELLP: "H = Hemolysis, EL = Elevated liver enzymes, LP = low platelets".

Figura 1 - Número e porcentagem de pacientes transferidas do Hospital Materno Infantil para as unidades de terapia intensiva, segundo doenças hipertensivas (causas obstétricas) em Goiânia, nos anos de 1999 a 2001

Encontramos nove outras causas obstétricas, como por exemplo: esteatose hepática $(n=4)$, hiperêmese gravídica grave $(n=3)$, um caso de óbito fetal com infecção intra-uterina e outro de abdome agudo após curetagem puerperal por restos placentários.

Dentre as causas não obstétricas $(17,4 \%$ das indicações), as cardiopatias representaram $26,7 \%$ $(n=4)$, sendo que houve ainda um caso de hipertensão arterial sistêmica e outro infeccioso (broncopneumonia com derrame pleural). Houve ainda nove outras causas não obstétricas, dentre as quais destacamos dois casos de apendicite aguda $(13,3 \%)$ e outros dois de asma grave $(13,3 \%)$. Houve um episódio de abdome agudo por perfuração de úlcera duodenal e outro por perfuração acidental do ceco, havendo ainda outros casos, como: anemia falciforme, carcinoma gástrico metastático e uma insuficiência hepática aguda em paciente portadora do vírus HIV.

Em relação ao momento das transferências, houve cinco casos em que as pacientes foram transferidas após episódio de abortamento, o que representou 5,8\% da nossa amostra. As transferências que ocorreram durante a gestação representaram $12,8 \%$ dos casos $(n=11)$, sendo que a idade gestacional média foi de 21,1 semanas, com desvio-padrão grande de 11,4 semanas. Verificamos que $81,4 \%$ das transferências foram realizadas no período puerperal $(\mathrm{n}=70)$, observando que, em 55 ocasiões $(78,6 \%)$, estas foram realizadas no mesmo dia da internação no Hospital Materno Infantil de Goiânia.

Quando avaliamos o tipo de parto, a cesariana representou a maioria $(72,4 \%)(55 / 76)$ e o parto vaginal correspondeu a $27,6 \%(21 / 76)$. Entre os partos vaginais, os espontâneos ocorreram em $61,9 \%$ das vezes $(n=13)$ e os induzidos em $38,1 \%$ $(\mathrm{n}=8)$, sendo que em relação aos partos cesáreos, a eletividade esteve presente em 96,3\% das ocasiões $(53 / 55)$.

Em nossa amostra, analisamos o desfecho materno por meio do número de dias de internação nas unidades de terapia intensiva, sendo que se considerarmos todos os grupos juntos, entre as 86 observações, percebe-se que a média foi de 5,1 dias e a mediana de 3,5 dias, com um desvio padrão de 6,3 dias (Tabela 2).

Tabela 2 - Dias de internação nas unidades de terapia intensiva por grupo etário.

\begin{tabular}{lccc}
\hline & Média & Dias de internação \\
\hline G $<19$ & 5,3 & 4,0 & 4,2 \\
G $19-35$ & 5,1 & 4,0 & 7,1 \\
G $>35$ & 3,2 & 3,0 & 1,1 \\
Todos os grupos & 5,1 & 3,5 & 6,3 \\
\hline
\end{tabular}

$\mathrm{G}<19$ : pacientes com idade inferior a 19 anos completos.

G 19-35: pacientes com idade entre 19 e 35 anos completos.

$G$ >35: pacientes com idade superior a 35 anos.

A mortalidade materna encontrada em nosso estudo foi elevada $(24,3 \%)$, sendo que este número reflete o perfil das causas de mortalidade materna no nosso país, uma vez que as doenças hipertensivas responsabilizaram-se por 52,9\% (9/ 17) das mesmas, destacando-se a eclâmpsia como a principal causa (5 casos), seguida pela síndrome HELLP (3 casos). As doenças hemorrágicas representaram três casos da nossa amostra, dando ênfase a dois casos de acretismo placentário que evoluíram para choque hipovolêmico e outro de abortamento com semelhante evolução. As causas infecciosas contabilizaram dois casos, sendo um de septicemia após abortamento e outro episódio de meningite após infecção puerperal. Destacaram-se ainda, entre as ditas outras causas obstétricas que levaram as pacientes ao óbito, dois casos de hiperêmese gravídica grave evoluindo para cetoacidose e outro de esteatose hepática com disfunção de múltiplos órgãos. 
Avaliando-se a mortalidade materna em relação aos quatro grupos de indicações obstétricas (sindromes hipertensivas, hemorrágicas, infecciosas e outras), percebemos que não houve diferença significante entre eles $(\mathrm{p}=0,815)$ (Tabela 3$)$. Outro aspecto analisado, em nossa pesquisa, foi a relação entre o tempo de internação nas UTI's (se menor ou maior que 48 horas) e a mortalidade materna, sendo que não observamos diferença, significante, entre as duas categorias. Ilustra-se que, na amostra aqui representada, 24 das 86 observações $(27,9 \%)$ tiveram período de internação nas UTI's inferior a 48 horas, sendo que, por outro lado, a maioria $(72,1 \%)$ permaneceu por tempo superior a 48 horas.

Tabela 3 - Número e porcentagem de mortes maternas, por indicações obstétricas, entre as pacientes transferidas do Hospital Materno Infantil para as unidades de terapia intensiva, em Goiânia, nos anos de 1999 a 2001.

\begin{tabular}{lccccc}
\hline Morte materna & \multicolumn{2}{c}{ Doenças $\mathbf{n}(\%)$} & & Total \\
& Hipertensivas & Hemorrágicas & Infecciosas & Outras & \\
\hline Sim & $9(21,9)$ & $3(21,4)$ & $2(33,3)$ & $3(33,3)$ & 17 \\
Não & $32(78,0)$ & $11(78,6)$ & $4(66,7)$ & $6(66,7)$ & 53 \\
Total & $41(58,5)$ & $14(20,0)$ & $6(85,7)$ \\
\hline
\end{tabular}

$\mathrm{p}=0,815$

Ainda dentro deste contexto mórbido, avaliamos o modo de apuração dos óbitos maternos, percebendo que em $70,6 \%$ dos casos $(n=12)$, este foi realizado por meio de exames laboratoriais e imaginológicos, sendo que em três situações $(17,6 \%)$ a propedêutica clínica foi soberana, tendo sido a necrópsia realizada em apenas dois casos (11,8\%).

Um dos desígnios deste estudo foi a análise da freqüência representativa da necessidade de cuidados intensivos em Obstetrícia a partir da RMI, obtida pela relação entre o número de transferências das pacientes obstétricas para as unidades de terapia intensiva e o número total de partos ocorridos, num determinado intervalo de estudo, por 1.000 partos.

Durante o período avaliado, houve 86 transferências para UTI's, sendo que no ano de 1999, ocorreram 32 (37,2\%). Em 2000, foram 23 (26,7\%) e, no ano seguinte, $31(36,1 \%)$ casos.

No universo de 4.560 partos ocorridos nos três anos desta pesquisa, 1.845 ocorreram em 1999, havendo ainda 1.405 e 1.310 nos anos seguintes de 2000 e 2001 . Quando calculamos a RMI nos três anos da pesquisa, obtemos 18,8/1.000 partos, sendo que em 1999 a RMI foi de 17,3 e nos anos de 2000 e $2001,16,3$ e 23,6 , respectivamente.

\section{Discussão}

Curioso é perceber que, a partir do início da década de 90, mais precisamente em 1992, época em que o estudo pioneiro sobre a necessidade de cuidados intensivos em Obstetrícia ${ }^{9}$ foi apresentado à comunidade científica mundial, pequeno número de publicações surgiu na literatura internacional a respeito do tema. É assunto preocupante porque indicadores das maternidades em nosso meio mostram, por vezes, uma falsa realidade: coeficientes de mortalidade materna baixos, como se estivéssemos nos chamados países de primeiro mundo ${ }^{10}$. Entretanto, observa-se, que com a transferência das pacientes obstétricas mais gravemente enfermas para as unidades de terapia intensiva, perde-se a evolução dos $\operatorname{casos}^{11} \mathrm{e}$ com isso os resultados ominosos são minimizados, uma vez que os especialistas da área não estão mais analisando e publicando o ocorrido ${ }^{12}$.

Percebemos a importância do número de nulíparas demonstrado nesta pesquisa, sendo que assim, ressaltamos a necessidade de atenção por parte dos tocólogos para este grupo especial de mulheres, geralmente jovens (média de 24,6 anos), que estão iniciando suas vidas reprodutivas. O desfecho determinado pelas síndromes hipertensivas induzidas pela gestação deve continuar preocupando o tocólogo, uma vez que acompanhamento pré-natal adequado, referenciamento oportuno dos casos mais graves para serviços terciários e conhecimento das condutas terapêuticas emergenciais dessas intercorrências são essenciais na tentativa de se minimizar este grave problema da Obstetrícia brasileira atual. Os eventos hemorrágicos no puerpério representam importante causa de necessidade de cuidados intensivos ${ }^{13}$, sendo que o pronto reconhecimento desses casos associado à existência de retaguarda de serviços com maior complexidade para o adequado acompanhamento são decisivos para a manutenção da vida dessas pacientes. 
Embora tenha ocorrido diminuição significativa nos casos infecciosos durante o ciclo gravídico-puerperal nas últimas décadas ${ }^{14}$, percebemos que práticas ilegais de abortamento e assistência inadequada ao parto ainda fazem parte da triste realidade assistencial do nosso país. É fundamental que haja o conhecimento por parte dos tocólogos das condutas diagnósticas e, sobretudo, terapêuticas, para idealizarmos o correto manejo dessas situações clínicas oriundas da gestação. Os números encontrados, em nossa amostra, nos fazem refletir sobre o momento em que estas transferências ocorrem no puerpério, visto que o predomínio do encaminhamento no puerpério imediato parece ter expressiva associação com a tentativa de resolução do fator obstétrico, desencadeador das disfunções sistêmicas, ainda no hospital terciário, antes da transferência das pacientes para as UTI's.

No presente estudo, verificamos elevado número de cesarianas eletivas, o que pode nos revelar tanto a gravidade das doenças obstétricas em questão, quanto a provável necessidade de interrupção rápida destas gestações críticas. A cesariana é um dos procedimentos cirúrgicos mais executados e debatidos na atualidade. Ao longo do tempo, sua realização levanta uma série de questões que refletem sobre o futuro reprodutivo da mulher. Embora o parto cesáreo esteja associado a elevados coeficientes de morbimortalidade materna quando comparado ao parto vaginal, interrupções bem indicadas e oportunas das gestações também podem contribuir de maneira importante para redução do risco de óbito materno-fetal ${ }^{15}$.

Ainda que sejam considerados os aspectos relativos à forma de organização da assistência obstétrica, à formação dos profissionais de saúde e à pressão por cesárea por parte das parturientes, é necessário que se avalie continuamente o verdadeiro impacto de tal procedimento sobre os indices de complicações materno-fetais.

O estudo da incidência e das causas de mortes maternas tem ocupado posição de destaque na área da saúde nos últimos tempos, sendo que a OMS e outras organizações internacionais têm recomendado aos governos dos países, sobretudo no "terceiro mundo", a adoção de medidas que visem à melhora de seu conhecimento e à diminuição de sua ocorrência ${ }^{16}$.

A incidência de óbitos observada em nosso estudo foi elevada $(24,3 \%)$, assim como os resultados obtidos pelos autores Royston e AbouZhar ${ }^{17}$. Em relação aos fatores determinantes das mortes maternas, prevalecem, no contexto do atendimento público brasileiro, as ditas causas obstétricas diretas em 74\% das vezes. Dados do Ministério da Saúde (2000) ressaltam, em ordem decrescente de freqüência, eclâmpsia, hemorragias, infecção puerperal e abortamento, como sendo as principais causas obstétricas diretas dos óbitos maternos ${ }^{18}$, o que se assemelha aos resultados obtidos em nossa presente pesquisa.

Atrash et al. ${ }^{19}$ acreditam que o problema fundamental é a chegada das pacientes graves, por vezes tardia, aos centros terciários de referência - e não carência de recursos humanos ou materiais dentro dos hospitais - para atender às complicações intercorrentes no puerpério, que ocasionam os decessos maternos. Em nossa amostra, houve predomínio $(72,1 \%)$ de internação nas UTI's por período superior a 48 horas, o que pode nos demonstrar a gravidade dos casos obstétricos transferidos, e nos fazer conjeturar os custos prováveis para o sistema de saúde ${ }^{20}$.

Existem importantes diferenças numéricas, quando comparamos a RMI obtida nesta pesquisa com a de outros autores (Graham e Luxton ${ }^{21}$ : $\mathrm{RMI}=1,0$; Monaco et al. ${ }^{5}: \mathrm{RMI}=2,5$; Cohen et al. ${ }^{22}$ : $\mathrm{RMI}=0,7$ ). Devido à raridade de óbitos maternos nos países desenvolvidos, é de grande importância que haja, nos países em desenvolvimento, estudos sobre os casos obstétricos mais graves e que demandam transferência para as unidades de terapia intensiva.

Muitos casos, sem dúvida, requerem procedimentos só disponiveis em hospitais de maior complexidade, mas na maioria das vezes o mais importante é instituir todos os cuidados possiveis em cada contexto, e só realizar o encaminhamento mediante condições seguras de remoção e com destino garantido ${ }^{23}$.

Seria ingênuo acreditarmos que se possa diminuir o número de óbitos maternos no Brasil aos níveis observados hoje nos países desenvolvidos, sem que ocorra substancial melhoria das condições de vida da população. Agora, com o estudo sobre o suporte intensivo obstétrico, poderemos identificar as grandes causas de transferências maternas para as UTI's e atuar para evitar o agravamento das intercorrências críticas, ou ainda, quando não for possível a transferência precoce dessas pacientes para unidades especializadas.

Assim, o trabalho em equipe interdisciplinar, entre obstetras e intensivistas, parece-nos a solução ideal, onde cada especialista poderia contribuir com suas noções diagnósticas ou terapêuticas para a evolução adequada e satisfatória destes casos graves. São necessárias mais trabalhos sobre este tema, prospectivos, com casuística ampliada, que comparem protocolos de transferências das pacientes obstétricas para as UTI's, com vistas a substituir o empirismo que ora reina em relação aos melhores caminhos para a tão desejada redução da mortalidade materna em nosso país. 


\section{ABSTRACT}

Objective: to evaluate the epidemiological and parturitional aspects of obstetric patients admitted to intensive care units (ICU), and analyze the frequency of intensive support needed by them.

Methods: observational and descriptive study of all obstetric patients' transfers to ICU from the Hospital Materno Infantil of Goiânia-Go, from January 1999 to December 2001. The analysis has included variables as maternal age, parity, obstetric and non-obstetric indications for ICU admissions, moment of transfer, mode of delivery, maternal death, and the frequency of ICU utilization per 1,000 deliveries (IDR imminent death ratio). The statistical analysis was performed by the $\chi^{2}$ test or the Fisher exact test and a significant difference was set at a level of $5 \%$.

Results: over the 36-month period analyzed, 86 pregnancyassociated ICU admissions were identified (among 4,560 deliveries). Of the 86 patients, $52.33 \%(n=45)$ were nulliparae and $63(73.26 \%)$ were between 19 and 35 years old. Hypertensive disorders accounted for 41 (57.75\%) of the admissions and hemorrhage for 14 (19.72\%). Eclampsia $(n=23)$, HELLP syndrome $(n=13)$ and premature abruptio placentae $(n=5)$ were the most common obstetric indications for ICU admissions. Maternal cardiac disorders accounted for 4 cases of non-obstetric indications. There was a predominance of postpartum transfers (82.35\%). Fifty-five $(72.37 \%)$ patients needed delivery by caesarian section. The average time spent in the UCI by those patients was 5.1 days. Maternal mortality found in this study was $24.29 \%$, hypertensive disorders being responsible for $52.94 \%$ (9/17) of all obstetric-associated deaths. There were no significant statistical differences $(p=0.81)$ regarding these obstetricassociated deaths and their causes (hypertensive disorders, hemorrhage or infections) or even regarding maternal deaths and duration of stay $(<$ or $>48$ hours) in the ICU $(p=0.08)$. The IDR found was 18.8 per 1,000 deliveries.

Conclusions: the need of intensive care estimated by IDR was 18.8 per 1,000 deliveries, the pregnancy-induced hypertension being responsible for the majority of the indications for maternal transfers.

KEYWORDS: Intensive care. Pregnancy-induced hypertension. Maternal death.

\section{Referências}

1. Benzecry R, Arkader J. Mortalidade materna. In: Benzecry R, Oliveira HC, Lengruber I, editores. Tratado de Obstetrícia FEBRASGO. $1^{\mathrm{a}}$ ed. Rio de Janeiro: Revinter; 2000. p.159-63.

2. Rezende J, Montenegro CAB, Belfort P. Mortalidade materna e perinatal. In: Rezende $\mathrm{J}$, editor. Obstetrícia. 8 ${ }^{a}$ ed. Rio de Janeiro: Guanabara Koogan; 1998. p.1317-25.

3. Mabie WC, Sibai BM. Treatment in an obstetric intensive care unit. Am J Obstet Gynecol 1990; 162:1-4.
4. Mantel GD, Buchmann E, Rees H, Pattinson RC. Severe acute maternal morbidity: a pilot study of a definition for a near-miss. Br J Obstet Gynaecol 1998; 105:985-90.

5. Monaco TJ Jr, Spielman FJ, Katz VL. Pregnant patients in the intensive care unit: a descriptive analysis. South Med J 1993; 86:414-7.

6. Belfort P. Mortalidade materna e qualidade de vida. GO Atual 2000; 6:3.

7. Viggiano MGC. Condutas em obstetrícia. Goiânia: Atheneu; 1994. p.15-21.

8. Rezende J, Montenegro CAB. O puerpério. In: Rezende J, editor. Obstetrícia Fundamental. $7^{\text {a }}$ ed. Rio de Janeiro: Guanabara Koogan; 1996. p.209-16.

9. Fitzpatrick C, Halligan A, McKenna P, Coughlan BM, Darling MR, Phelan D. Near miss maternal mortality (NMM). Ir Med J 1992; 85:37.

10.Viggiano MGC, Viggiano MB. O termo near-miss mortality aplicado à realidade obstétrica brasileira. GO Atual 2000; 9:29-34.

11.Thibault G, Mulley AG, Barnett GO, et al. Medical intensive care: indications, interventions, and outcomes. N Engl J Med 1995; 302:938-42.

12.Finn WF. Maternal welfare: Nassau County, New York, 1957-1981. Obstet Gynecol Surv 1991; 39:127-33.

13.Nasrat HA, Youssef MH, Marzoogi A, Talab F. Nearmiss obstetric morbidity in an inner city hospital in Saudi Arabia. East Mediterr Health J 1999; 5:717-26.

14.Faúndes A, Cecatti JG, Bacha AM, Pinotti JA. Intervenções para a redução da mortalidade materna. Rev Paul Med 1989; 107:47-52.

15. Grimes DA. The morbidity and mortality of pregnancy: still risky business. Am J Obstet Gynecol 1994; 170:1489-94.

16.Drife JO. Maternal "near miss” reports? BMJ 1993; 307:1087-9.

17. Royston E, AbouZahr C. Measuring maternal mortality. Br J Obstet Gynaecol 1992; 99:540-3.

18. Ministério da Saúde. Secretaria de Políticas de Saúde. Área Técnica de Saúde da Mulher. Urgências e emergências maternas: guia para diagnóstico e conduta em situações de risco de morte materna. Brasília; 2000. p.4-12.

19.Atrash HK, Alexander S, Berg CJ. Maternal mortality in developed countries: not just a concern of the past. Obstet Gynecol 1995; 86:700-5.

20.Clark S, Hankins G, Phelan J. Tratamento intensivo em obstetrícia. $2^{\text {a }}$ ed. São Paulo: Ed. Santos; 2001. p.4.

21.Graham SG, Luxton MC. The requirement for intensive care support for the pregnant population. Anaesthesia 1989; 44:581-4.

22. Cohen J, Singer P, Kogan A, Hod M, Bar J. Course and outcome of obstetric patients in a general intensive care unit. Acta Obstet Gynecol Scand 2000; 79:846-50.

23.Wright D, Mackenzie SJ, Buchan I, Cairns CS, Price L. Critical incidents in the intensive therapy unit. Lancet 1991; 338:676-8. 